\title{
Clinical MR Spectroscopy: Techniques and Applications
}

\author{
P.B. Barker, A. Bizzi, N. De Stefano, R. Gullapalli, and D.D.M. Lin, eds.
}

New York, NY: Cambridge University Press, 2010, 264 pages, $\$ 120$

MR spectroscopy (MRS) is an analytic technique widely used in chemistry for analyzing the structure of compounds and the composition of mixtures of compounds. Compounds are identified by their unique spectra, based on chemical shifts and coupling constants. MRS allows the noninvasive measurement of selected biologic compounds in vivo. Major technical advances have occurred in MRS over the last several decades, including superconducting magnets and Fourier transform for signal processing. Feasibility was first demonstrated in humans in the mid1980s, and much experience with MRS has accumulated in both research and clinical applications. Nearly all MRI scanners have the capability of performing MRS, and MRS techniques continue to improve even after 2 decades of development. Despite this considerable research effort and the unique biochemical information provided, only limited integration of MRS into clinical practice has occurred, for multiple reasons including nonstandardization of acquisition and analysis protocols, limited vendor support, difficult interpretation, limited perceived added value above conventional MRI, and lack of reimbursement. However, in vivo MRS is increasingly being used in clinical practice, particularly for neurologic disorders. Proton spectroscopy of the human brain is most widely used, but other organ systems such as breast and prostate, and other nuclei including ${ }^{31} \mathrm{P}$ and ${ }^{13} \mathrm{C}$, have been studied. In the brain, compounds of key importance measured by MRS include $\mathrm{N}$-acetyl aspartate (located predominantly in neurons), choline, myoinositol (located primarily in glial cells), creatine, lactate, glutamate, and glutamine.

This book was written by leading MRS experts, and it is an invaluable guide for anyone interested in in vivo MRS, including radiologists, nuclear physicians, neurologists, neurosurgeons, oncologists, and medical researchers. It gives the reader a solid basis for understanding both the techniques and the applications of clinical MRS. The book is organized into 14 chapters. Chapter 1 introduces in vivo MRS, and chapter 2 discusses pulse sequences and protocol design. Chapter 3 addresses spectral analysis methods, quantitation, and common artifacts, and chapter 4 handles normal regional variations, particularly brain development and aging. The rest of the chapters discuss MRS findings in brain tumors; in stroke and hypoxic-ischemic encephalopathy; in infectious, inflammatory and demyelinating lesions; in epilepsy; in neurodegenerative diseases; in traumatic brain injury; in cerebral metabolic disorders; in prostate cancer; in breast cancer; and in musculoskeletal diseases. Each chapter begins with key points and ends with recommendations and a conclusion. References are updated and useful.

The aim of this book is to serve as a practical reference work that covers all aspects of in vivo human spectroscopy for clinical purposes. The book explains physical principles and provides a comprehensive and perceptive review of clinical applications. Also discussed are the limitations of MRS, such as its low spatial resolution when compared with MRI, common artifacts, and diagnostic pitfalls. More widespread adoption of MRS into the clinic will lead to better diagnoses and improved outcomes for individual patients. There are 140 figures, which are clear and have detailed legends, and 7 tables that are helpful for readers. The index is convenient and useful. I highly recommend this book to trainees and practitioners in medical physics, radiology, nuclear medicine, oncology, neurology, and cardiology.

\section{E. Edmund Kim}

M.D. Anderson Cancer Center

1515 Holcombe Blvd., Unit 1264

Houston, TX 77030

E-mail: ekim@di.mdacc.tmc.edu 